\title{
Synthesis, Characterization and Primary Antimicrobial, Antifungal Activity Evaluation of Schiff bases of 4-Chloro-(3-substituted- phenylimino) - methyl-[2H] - chromene-2-one
}

\author{
AMIT KASABE*, VIJAY MOHITE, \\ JAYESH GHODAKE and JAYDEEP VIDHATE \\ Department of Pharmaceutical Chemistry, \\ Nandha College of Pharmacy, Erode, Tamilnadu, India. \\ amit9590@gmail.com
}

Received 28 July 2009; Revised 19 September; Accepted 15 November 2009

\begin{abstract}
Coumarins show promising antimicrobial and antifungal activity, this prompted to us to synthesize new derivatives of coumarins. 4-Chloro-2-oxo- $2 \mathrm{H}$ chromene-3-carbaldehyde was reacted with substituted aniline and rectified spirit to obtain a new series of Schiff bases of 4-chloro-(3-substituted-phenylimino) -methyl$[2 H]$-chromene-2-one (3a-i). Their structures were confirmed by IR, ${ }^{1} \mathrm{H}$ NMR spectral data. The synthesized compounds were investigated for antimicrobial and antifungal activities. The data reported showed that the effect of variation in chemical structure was rather unpredictable. Seldom did a particular structural modification lead to uniform alteration in activity in all tests. The substitution which appeared to be most important for high order of activity in the number of test was the 3-chloro phenylimino group. The observed antimicrobial and antifungal activities are attributed to the substitution at $3^{\text {rd }}$ position of phenylimino moiety.
\end{abstract}

Keywords: Schiff base, Antimicrobial Activity, Antifungal Activity, 4-Chloro-3-coumarinaldehyde.

\section{Introduction}

Coumarins have a long history of having number of pharmacological activities such as anticoagulant, antithrombotic, antimutagenic, vasodilator, LOX and CLOX inhibitors and it can also used in treatment of edema ${ }^{1-3}$. The recent success of coumarins as antimicrobial and antifungal has further highlighted the importance of this class in medicinal chemistry ${ }^{4-10}$. A systematic investigation of this class of compound revealed that coumarin derivatives containing pharmacophore agent plays an important role in medicinal chemistry. It has been reported that naturally occurring coumarin derivatives such as $2 \mathrm{H}$-1-benzopyran-2-one, 4hydroxycoumarins shows different pharmacological activities which are clinically important. As literature survey shows that, Schiff bases too have antimicrobial and antifungal activity this promoted to us to synthesize new derivatives of coumarins ${ }^{11,12}$. In view of these above, an 
attempt has been undertaken for the synthesis of new Schiff bases of 4-chloro-(3-substitutedphenylimino)-methyl-[2H]-chromene-2-one, possessing moderate biological activities. The synthesized compounds were tested for their possible antimicrobial and antifungal activities.

\section{Experimental}

Melting points were estimated with Veego (VMP-D) melting point apparatus in open capillaries and are uncorrected. Purity of compounds was checked by TLC. IR spectra $\left(\mathrm{KBr}, \mathrm{cm}^{-1}\right)$ were recorded on a Shimadzu 8400 -SFT-IR spectrometer. ${ }^{1} \mathrm{H}$ NMR $\left(\mathrm{CDCl}_{3}\right)$ on a VarianMercury-300 MHz spectrometer using TMS as an internal reference (chemical shift in $\delta \mathrm{ppm}$ ).

\section{Preparation of 4-hydroxycoumarin (1)}

To a mixture of $o$-hydroxyacetophenone $(10.0 \mathrm{~mol})$, sodium $(25.0 \mathrm{~mol})$ and dietylcarbonate (30.0 mol), mixed well and heated at $160{ }^{\circ} \mathrm{C}$ with constant stirring. The mixture was diluted with xylene $(30 \mathrm{~mL})$ and further heated at $160{ }^{\circ} \mathrm{C}$ for $1 \mathrm{~h}$ and then the mixture was poured into cold water $(400 \mathrm{~mL})$. $\mathrm{NaOH}(2 \mathrm{~N})$ was used to make the mixture alkaline and mixture was stirred with diethyl ether. After which the aqueous phase was collected and then acidified with $\mathrm{HCl}(6 \mathrm{~N})$. Product was filtered, washed with ice cold water and dried in vacuum desiccator.

\section{Preparation of 4-chloro-2-oxo-2H-chromene-3-carbaldehyde (2)}

To a stirred mixture of 4-hydroxycoumarin $(0.06 \mathrm{~mol})$ in anhydrous DMF ( $0.6 \mathrm{~mol})$ was added to which $\mathrm{POCl}_{3}(0.18 \mathrm{~mol})$ was added drop wise at $-10^{\circ}$ to $-5^{\circ} \mathrm{C}$. Mixture was then stirred for $1 \mathrm{~h}$ at room temperature and heated and stirred for $2 \mathrm{~h}$ at $60^{\circ} \mathrm{C}$. After the reaction was completed, the mixture was kept overnight at $0{ }^{\circ} \mathrm{C}$. The separated pale yellow solid was collected by filtration and washed successively with $\mathrm{Na}_{2} \mathrm{CO}_{3}$ solution (5\%) and water and was dried in air.

\section{Preparation of Schiff base of 4-chloro-3-coumarinaldehyde (3)}

4-Chloro-3-Coumarinaldehyde, $(0.005 \mathrm{~mol})$ of aniline and rectified spirit, was reflux for 20 min. Water was then added. The oil that separated was induced to crystallize by rubbing with glass rod and the solid was collected by filtration. After washing well with cold ethanol (88\%), the crude Schiff base was dried and recrystallised from aq. methanol. Compounds (3ai) were prepared similarly by using different arylaldehydes. Their melting points, $\%$ yields and molecular formula are given in Table 1.

Table 1. Characterization data of compounds (3a-i).

\begin{tabular}{|c|c|c|c|c|c|c|}
\hline $\begin{array}{c}\text { Compd } \\
\text { No. }\end{array}$ & $\mathrm{R}$ & $\begin{array}{l}\text { Molecular } \\
\text { formula }\end{array}$ & $\begin{array}{c}\text { Molecular } \\
\text { weight }\end{array}$ & $\begin{array}{l}\text { Melting } \\
\text { point, }{ }^{0} \mathrm{C}\end{array}$ & $\begin{array}{c}\mathrm{R}_{\mathrm{f}} \\
\text { Value }\end{array}$ & $\begin{array}{c}\text { Percentage } \\
\text { yield, } \%\end{array}$ \\
\hline $\mathbf{3 a}$ & $2-\mathrm{NO}_{2}$ & $\mathrm{C}_{16} \mathrm{H}_{9} \mathrm{ClN}_{2} \mathrm{O}_{4}$ & 328.71 & $295-296$ & 0.52 & 76.68 \\
\hline $3 \mathbf{b}$ & $4-\mathrm{NO}_{2}$ & $\mathrm{C}_{16} \mathrm{H}_{9} \mathrm{ClN}_{2} \mathrm{O}_{4}$ & 328.71 & $162-164$ & 0.63 & 67.48 \\
\hline $3 c$ & $4-\mathrm{Cl}$ & $\mathrm{C}_{16} \mathrm{H}_{9} \mathrm{Cl}_{2} \mathrm{NO}_{2}$ & 318.15 & $167-168$ & 0.62 & 75.94 \\
\hline 3d & $3-\mathrm{OCH}_{3}$ & $\mathrm{C}_{17} \mathrm{H}_{12} \mathrm{ClNO}_{3}$ & 313.74 & $167-168$ & 0.65 & 73.71 \\
\hline $3 e$ & $4-\mathrm{OCH}_{3}$ & $\mathrm{C}_{17} \mathrm{H}_{12} \mathrm{ClNO}_{3}$ & 313.74 & $126-127$ & 0.58 & 60.89 \\
\hline $3 f$ & $2-\mathrm{OCH}_{3}$ & $\mathrm{C}_{17} \mathrm{H}_{12} \mathrm{ClNO}_{3}$ & 313.74 & $119-121$ & 0.64 & 70.94 \\
\hline $3 g$ & $3-\mathrm{CH}_{3}$ & $\mathrm{C}_{17} \mathrm{H}_{12} \mathrm{ClNO}_{2}$ & 297.74 & $165-170$ & 0.58 & 64.18 \\
\hline $3 \mathbf{h}$ & $4-\mathrm{CH}_{3}$ & $\mathrm{C}_{17} \mathrm{H}_{12} \mathrm{ClNO}_{2}$ & 297.74 & 203-205 & 0.61 & 67.56 \\
\hline $3 \mathbf{i}$ & $\mathrm{H}$ & $\mathrm{C}_{16} \mathrm{H}_{10} \mathrm{ClNO}_{2}$ & 283.71 & $179-180$ & 0.68 & 78.01 \\
\hline
\end{tabular}


1: m.p. $213-215{ }^{0} \mathrm{C}$, yield: $93.4 \%$, IR ( $\mathrm{KBr}$ in $\left.\mathrm{cm}^{-1}\right)$ : 3421(O-H str.), 2885, 3003, 3073 (C-H str.), $1647\left(\mathrm{C}=\mathrm{O}\right.$ str.): $\mathrm{R}_{\mathrm{f}}$ : 0. 8. 2: m.p. $120-122^{\circ} \mathrm{c}$, yield: $72 \%$, IR $\left(\mathrm{KBr}\right.$ in $\left.\mathrm{cm}^{-1}\right)$ : 3061.13, 2918.40 (C-H str.) 1741.78 (C=O str.); 758.05(C-Cl str). ${ }^{1} \mathrm{H}$ NMR ( $\delta$ ppm) $\left(\mathrm{CDCl}_{3}\right): 7.3-8.13(4 \mathrm{H}, \mathrm{s}, \mathrm{Ar}-\mathrm{H}), 10.34(\mathrm{~s}, 1 \mathrm{H}, \mathrm{CHO}) . \mathrm{R}_{\mathrm{f}}: 0.83$

3a: $\left(\mathrm{R}=2-\mathrm{NO}_{2}\right)$ m.p. $295-296{ }^{\circ} \mathrm{C}$, yield: $76.68 \%$, IR $\left(\mathrm{KBr}\right.$ in $\left.\mathrm{cm}^{-1}\right): 3078.49$ (C-H str.), 1739.85(C=O str.), $1602.90(\mathrm{C}=\mathrm{N}$ str. $), \quad 796.12(\mathrm{C}-\mathrm{Cl}$ str. $) .{ }^{1} \mathrm{H}$ NMR $(\delta \mathrm{ppm})\left(\mathrm{CDCl}_{3}\right)$ : 8.758(s,1H,-CH=N-), 7.601(t,2H,Ar-H), 7.828-7.990(d,1H,Ar-H), 8.192(d,1H,Ar-H), 7.4557.579(m,2H,Ar-H).

3b: $\left(\mathrm{R}=-4-\mathrm{NO}_{2}\right)$ : m.p. $162-164{ }^{0} \mathrm{C}$, yield: $67.48 \%$, IR $\left(\mathrm{KBr}\right.$ in $\left.\mathrm{cm}^{-1}\right): 3082.35,3051.49$ (C-H str.), 1716.70(C=O str.), 1519.96(C=N str.), 758.05(C-Cl str.): ${ }^{1} \mathrm{H}$ NMR $(\delta$ ppm $)$ $\left(\mathrm{CDCl}_{3}\right): 8.758(\mathrm{~s}, 1 \mathrm{H},-\mathrm{CH}=\mathrm{N}-), 7.601(\mathrm{t}, 2 \mathrm{H}, \mathrm{Ar}-\mathrm{H}), 7.828-7.990(\mathrm{~d}, 1 \mathrm{H}, \mathrm{Ar}-\mathrm{H}), 8.192(\mathrm{~d}, 1 \mathrm{H}, \mathrm{Ar}-$ $\mathrm{H}), 7.455-7.579(\mathrm{~m}, 2 \mathrm{H}, \mathrm{Ar}-\mathrm{H})$.

3c: $(\mathrm{R}=4-\mathrm{Cl})$ : m.p. $167-168{ }^{\circ} \mathrm{C}$, yield: $75.94 \%$, IR $\left(\mathrm{KBr}\right.$ in $\left.\mathrm{cm}^{-1}\right): \quad 3117,3080,3063$, (C-H str.), 1693(C=O str.), 1541.18(C=N str.), 717.54(C-Cl str.). ${ }^{1} \mathrm{H}$ NMR $(\delta \mathrm{ppm})\left(\mathrm{CDCl}_{3}\right)$ : 8.758(s,1H,-CH=N-), 7.601(t,2H,Ar-H), 7.828-7.990(d,1H,Ar-H), 8.192(d,1H,Ar-H), 7.4557.579(m,2H,Ar-H).

3d: $\left(\mathrm{R}=3-\mathrm{OCH}_{3}\right)$ : m.p. $200-201{ }^{0} \mathrm{C}$, yield: $73.71 \%$, IR $\left(\mathrm{KBr}\right.$ in $\left.\mathrm{cm}^{-1}\right): \quad 3066.92,3016.77$ $(\mathrm{C}-\mathrm{H}), 1739(\mathrm{C}=\mathrm{O}), 1473.66(\mathrm{C}=\mathrm{N}), 759.98(\mathrm{C}-\mathrm{Cl}):{ }^{1} \mathrm{H}$ NMR $(\delta \mathrm{ppm})\left(\mathrm{CDCl}_{3}\right): 8.758(\mathrm{~s}, 1 \mathrm{H},-$ $\mathrm{CH}=\mathrm{N}-), \quad$ 7.601 $(\mathrm{t}, 2 \mathrm{H}, \mathrm{Ar}-\mathrm{H}), \quad$ 7.828-7.990(d,1H,Ar-H), $\quad$ 8.192(d,1H,Ar-H), 7.579(m,2H,Ar-H), 3.431(s, $\left.1 \mathrm{H}, \mathrm{OCH}_{3}\right)$.

3e: $\left(\mathrm{R}=4-\mathrm{OCH}_{3}\right)$ : m.p. $126-127{ }^{0} \mathrm{C}$, yield: $60.89 \%$, IR $\left(\mathrm{KBr}\right.$ in $\left.\mathrm{cm}^{-1}\right): 3078,2924(\mathrm{C}-\mathrm{H}$ str.), $1714(\mathrm{C}=\mathrm{O}$ str. $), 1518.03(\mathrm{C}=\mathrm{N}$ str. $), 754.19(\mathrm{C}-\mathrm{Cl}$ str. $):{ }^{1} \mathrm{H}$ NMR $(\delta \mathrm{ppm})\left(\mathrm{CDCl}_{3}\right)$ : 8.758 (s,1H,-CH=N-), 7.601(t,2H,Ar-H), 7.828-7.990(d,1H,Ar-H), 8.192(d,1H,Ar-H), 7.455-7.579(m,2H,Ar-H), 3.431( $\left.\mathrm{s}, 1 \mathrm{H}, \mathrm{OCH}_{3}\right)$.

3f: $(\mathrm{R}=2-\mathrm{OCH})$ : m.p. $119-121{ }^{\circ} \mathrm{C}$, IR $\left(\mathrm{KBr}\right.$ in $\left.\mathrm{cm}^{-1}\right)$ : 3061.13(C-H), $1739.85(\mathrm{C}=\mathrm{O})$, 1602.50(C=N), $769.98(\mathrm{C}-\mathrm{Cl}):{ }^{1} \mathrm{H} \quad \mathrm{NMR}(\delta \mathrm{ppm}) \quad\left(\mathrm{CDCl}_{3}\right): \quad 8.758(\mathrm{~s}, 1 \mathrm{H},-\mathrm{CH}=\mathrm{N}-)$, 7.601(t,2H,Ar-H), 7.828-7.990(d,1H,Ar-H), 8.192(d,1H,Ar-H), 7.455-7.579(m,2H,Ar-H).

3g: $\left(\mathrm{R}=3 \mathrm{CH}_{3}\right)$ : m.p. $165-170{ }^{\circ} \mathrm{C}$, yield: $64.18 \%$, IR $\left(\mathrm{KBr}\right.$ in $\left.\mathrm{cm}^{-1}\right): 3340.82,3061.13(\mathrm{C}-$ $\mathrm{H}), 1730.99(\mathrm{C}=\mathrm{O}), 1504.53(\mathrm{C}=\mathrm{N}), 754.19(\mathrm{C}-\mathrm{Cl}):{ }^{1} \mathrm{H} \mathrm{NMR}(\delta \mathrm{ppm})\left(\mathrm{CDCl}_{3}\right): 8.758(\mathrm{~s}, 1 \mathrm{H},-$ $\mathrm{CH}=\mathrm{N}), \quad$ 7.601(t,2H,Ar-H), 7.828-7.990(d,1H,Ar-H), 8.192(d,1H,Ar-H), 7.579(m,2H,Ar-H), 2.441(s,3H, $\left.\mathrm{CH}_{3}\right)$.

3h: $\left(\mathrm{R}=4 \mathrm{CH}_{3}\right)$ : m.p. $179-180{ }^{\circ} \mathrm{C}$, yield: $78.01 \%$, IR $\left(\mathrm{KBr}\right.$ in $\left.\mathrm{cm}^{-1}\right): 3061.13,2677.29(\mathrm{C}-\mathrm{H})$, 1741 $(\mathrm{C}=\mathrm{O}), 1500.67(\mathrm{C}=\mathrm{N}), 780.30(\mathrm{C}-\mathrm{Cl}):{ }^{1} \mathrm{H}$ NMR $(\delta \mathrm{ppm})\left(\mathrm{CDCl}_{3}\right): 8.758(\mathrm{~s}, 1 \mathrm{H},-\mathrm{CH}=\mathrm{N}-)$, 7.601(t,2H,Ar-H), 7.828-7.990(d,1H,Ar-H), 8.192(d,1H,Ar-H), 7.455-7.579(m,2H,Ar-H).

3i: $(\mathrm{R}=\mathrm{H})$ : m.p. $203-205{ }^{\circ} \mathrm{C}$, yield: $65.17 \%$, IR $\left(\mathrm{KBr}\right.$ in $\left.\mathrm{cm}^{-1}\right): 3117.07,3063(\mathrm{C}-\mathrm{H})$, 1693.56(C=O), $1541.18(\mathrm{C}=\mathrm{N}), 717.54(\mathrm{C}-\mathrm{Cl}):{ }^{1} \mathrm{H}$ NMR $(\delta \mathrm{ppm})\left(\mathrm{CDCl}_{3}\right): 8.758(\mathrm{~s}, 1 \mathrm{H},-$ $\mathrm{CH}=\mathrm{N}), 7.601(\mathrm{t}, 2 \mathrm{H}, \mathrm{Ar}-\mathrm{H})$, 7.828-7.990(d,1H,Ar-H), 8.192(d,1H,Ar-H), 7.455-7.579(m,2H, Ar-H), 2.441(s,3H, $\left.\mathrm{CH}_{3}\right)$.

Statistical analysis

Data were analyzed by one -way ANOVA followed by Dunnett's $t$-test using computerized Graph Pad Instat version 3.05.

Antibacterial activity

In vitro antimicrobial study was carried on Muller hinton agar (Hi-media) plates $\left(37^{\circ} \mathrm{C}, 24 \mathrm{~h}\right)$ by agar diffusion cup plate method ${ }^{13}$. All the compounds were screened for antimicrobial activity at $50,100,150,200 \mu \mathrm{g} / \mathrm{mL}$ concentration against the following bacterial strains 
Staphylococcus aureus, Bacillus subtilis, Escherichia coli. Minimum inhibitatory concentration (MIC) of the test solution was determined by using broth dilution method. Antifungal activity was tested on Muller hinton agar (Hi-media) plates $\left(37{ }^{\circ} \mathrm{C}, 24 \mathrm{~h}\right)$ by agar diffusion cup plate method ${ }^{13}$, against Candida albicans and Aspergillus niger at conc. Level at 50,100,150,200 $\mu \mathrm{g} / \mathrm{mL}$. Amoxycillin and Fluconazole were used as standard for antimicrobial and antifungal activity under similar conditions. DMSO was used as solvent control for both antimicrobial and antifungal activities. The results are presented in Table 2.

Table 2. Antimicrobial and antifungal activity of compounds 3(a-i).

\begin{tabular}{|c|c|c|c|c|c|c|c|}
\hline \multirow{3}{*}{ S. No. } & \multirow{3}{*}{$\begin{array}{l}\text { Compound } \\
\text { name }\end{array}$} & \multirow{3}{*}{$\begin{array}{c}\text { Concentration } \\
\mu \mathrm{g} / 10 \mu \mathrm{L}\end{array}$} & \multicolumn{5}{|c|}{ Zone of inhibition in $\mathrm{mm}$} \\
\hline & & & \multicolumn{3}{|c|}{ Bacteria } & \multicolumn{2}{|c|}{ Fungi } \\
\hline & & & E.Coli & B.Subtilis & S.Aureus & A.Niger & C.Albicans \\
\hline \multirow[t]{4}{*}{1.} & $3 \mathbf{a}$ & 50 & - & - & - & - & - \\
\hline & & 100 & 13 & 11 & 12 & 9 & 7 \\
\hline & & 150 & 14 & 16 & 18 & 14 & 11 \\
\hline & & 200 & 25 & 22 & 24 & 19 & 15 \\
\hline \multirow[t]{4}{*}{2.} & $\mathbf{3 b}$ & 50 & - & - & - & - & - \\
\hline & & 100 & 12 & 11 & 10 & 8 & 8 \\
\hline & & 150 & 18 & 17 & 15 & 12 & 12 \\
\hline & & 200 & 24 & 23 & 21 & 17 & 16 \\
\hline \multirow[t]{4}{*}{3.} & $3 c$ & 50 & 8 & 7 & 7 & - & - \\
\hline & & 100 & 16 & 14 & 15 & 10 & 10 \\
\hline & & 150 & 24 & 21 & 22 & 16 & 15 \\
\hline & & 200 & 32 & 28 & 30 & 22 & 21 \\
\hline \multirow[t]{4}{*}{4.} & $3 d$ & 50 & - & - & - & - & - \\
\hline & & 100 & 10 & 11 & 10 & 7 & 7 \\
\hline & & 150 & 15 & 17 & 15 & 11 & 10 \\
\hline & & 200 & 21 & 23 & 20 & 15 & 14 \\
\hline \multirow[t]{4}{*}{5.} & $3 e$ & 50 & - & - & - & - & - \\
\hline & & 100 & 8 & 10 & 9 & 7 & 10 \\
\hline & & 150 & 12 & 15 & 14 & 10 & 15 \\
\hline & & 200 & 17 & 21 & 19 & 14 & 20 \\
\hline \multirow[t]{4}{*}{6.} & 3f & 50 & - & - & - & - & - \\
\hline & & 100 & 9 & 10 & 8 & 7 & 9 \\
\hline & & 150 & 13 & 15 & 12 & 11 & 14 \\
\hline & & 200 & 18 & 20 & 17 & 15 & 19 \\
\hline \multirow[t]{4}{*}{7.} & $3 g$ & 50 & - & - & - & - & - \\
\hline & & 100 & 11 & 12 & 13 & 9 & 8 \\
\hline & & 150 & 16 & 18 & 19 & 13 & 12 \\
\hline & & 200 & 22 & 24 & 26 & 18 & 17 \\
\hline \multirow[t]{4}{*}{8.} & $3 h$ & 50 & - & - & - & - & - \\
\hline & & 100 & 10 & 11 & 12 & 9 & 8 \\
\hline & & 150 & 15 & 16 & 18 & 14 & 12 \\
\hline & & 200 & 21 & 22 & 24 & 19 & 16 \\
\hline \multirow[t]{4}{*}{9.} & $3 \mathbf{i}$ & 50 & - & - & - & - & - \\
\hline & & 100 & 9 & 7 & 8 & 7 & \\
\hline & & 150 & 13 & 10 & 12 & 9 & 8 \\
\hline & & 200 & 18 & 14 & 17 & 13 & 11 \\
\hline
\end{tabular}




\section{Results and Discussion}

Spectral characterization (IR, ${ }^{1} \mathrm{H}$ NMR) of final compound is provided in this section. The ${ }^{1} \mathrm{H}$ NMR spectrum of all the Schiff base compound is in between 7-8.758 (Aromatic $\mathrm{H}$ ) and 2.65-3.82 $\left(\mathrm{S}_{\text {of }} \mathrm{CH}_{3}\right)$. The characterization data of synthesized compounds is provided in Table 1. Biological results are reported in Table 2, which also included results of standard drugs. There was moderate antimicrobial and antifungal activity associated with synthesized compounds. The significant changes in potency resulted from minor change in chemical structure is shown in Table 2.

Synthetic scheme<smiles>CC(=O)c1ccccc1O</smiles><smiles>[CH2+]CCOC(=O)OCC(C)[14CH3]</smiles><smiles>O=c1cc(O)c2ccccc2o1</smiles>

(1)

$\mathrm{POCl}_{3},-5$ to $-10{ }^{\circ} \mathrm{c}$<smiles>[R]c1cccc(N=Cc2c(Cl)c3ccccc3oc2=O)c1</smiles>

Substituted anilines<smiles>O=Cc1c(Cl)c2ccccc2oc1=O</smiles>

(3a-i)

$$
\begin{gathered}
\mathrm{R}=2-\mathrm{NO}_{2}(\mathbf{3 a}), 4-\mathrm{NO}_{2}(\mathbf{3 b}), 4-\mathrm{Cl}(\mathbf{3 c}), 3-\mathrm{OCH}_{3}(\mathbf{3 d}), \\
4-\mathrm{OCH}_{3}(\mathbf{3 e}), 2-\mathrm{OCH}_{3}(\mathbf{3 f}), 3-\mathrm{CH}_{3}(\mathbf{3 g}), 4-\mathrm{CH}_{3}(\mathbf{3 h}), \mathrm{H}(\mathbf{3 i}),
\end{gathered}
$$

\section{Scheme 1.}

\section{Conclusion}

A total of 9 compounds were synthesized and screened for their antimicrobial activity against S. aureus, B. subtilis and E.coli and antifungal activity against A. niger and C.albicans. The MIC of all compounds was determined. The compounds were found to have moderate antimicrobial activity

The observed antimicrobial and antifungal activities are attributed to the substitution of 3-chloro, 3-methoxy group at 3 position of phenylimino ring of synthesized compounds. Obviously, the comparative evaluation of active compounds will require further studies; the data reported in this article may be helpful guide for the medicinal chemist who is working in this area.

\section{Acknowledgment}

The authors would like to express their gratitude and thanks to the Dept. of Pharmaceutical Chemistry, Nandha College of Pharmacy, Erode for necessary facilities for this research work and to Chandra Diagnostic Lab, Salem for carrying out microbiological screening. 


\section{References}

1. Principles of Medicinal Chemistry: Edited by William O Foye, 1989, 382.

2. Kimura Y, Okuda H, Arichi S, Baba K and Kozawa M, Biochim Biophys Acta, 1985, 834, 224.

3. Hoffmanova J, Kozubik A, Dusek L and Pachernik J, Eur J Pharmacol., 1998, 350, 273.

4. Laurent S, Fabienne C and Michel k, Tetrahydron letters, 2000, 41, 1543-1547.

5. Zalfiqar A and Nasim H, Indian J Chem., 2007, 46B, 1322-1327.

6. Bernadette S, Denis A and Kevin k, Inorganica Chemica Acta, 2006, 359, 3976-3984.

7. Bernadette S, Denis A and Kevin k, J Inorg Biochem., 2007, 101, 1108-1119.

8. Kumar Sanjeev S Lamani, Oblennavar Kotresh, Mohammedshafi A Phaniband, Jagannath C. Kadakol, E Journal of Chemistry, 2009, 6(S1), S239-S246.

9. Khan I and Kulkarni M, Indian J Chem., 1999, 38B, 491- 494.

10. Sreenivasulu B, Sundaramurthy V and Subbarao N, J Indian Chem Soc., 1974, 51,488.

11. Michael J Hearn and Michael H Cynamon, J Antimicrobial Chemotherapy, 2004, 12, 2413-2426.

12. Ashis kumar Nanda, Subarna Ganguli and Ranadhir Chakraborty, Molecule, 2007, 12, 2413-2426.

13. British Pharmacopoeia 2007, Appendix XVIB, Crown copyright, London, 2006.

14. Braccio M, Grossi G, Roma G, Signorello M and Leancini G, Eur J Med Chem., 2004, 39, 397-409.

15. Rajanna K and Saiprakash P, Int J Chem Kinet., 1996, 28, 865-872.

16. Furniss B and Hannaford A, Smith P W G.Vogel's Textbook of Practical Organic Chemistry, 1996, 5, 905.

17. Khan I, Kulkarni M and Chung-Ming Sun, Eur J Med Chem., 2005, 40, 1168-1172.

18. Hugo W and Russell A, Pharmaceut Microbiol., 1987, 4, 265.

19. Chandan Adhikary, Dasarath Mal, Ken-Ichi Okamoto, Siddhartha Chaudhuri and Subratanath Koner, Polyhedron, 2006, 11, 2191-2197.

20. Rufen Zhang, Qingfeng Wang, Qianli Li and Chunlin Ma, Inorganica Chimica Acta, 2009, 362, 2762-2769.

21. Thahira B.S.A. Ravoof, Karen A. Crouse, M. Ibrahim M. Tahir, Andrew R. Cowley, M. Akbar Ali, Polyhedron, 2007, 1159-1165.

22. Prashanthi Y, Kiranmai K, Subhashini N J P, Shivraj, Spectrochimica Acta, 2008, 70A, 30-35. 


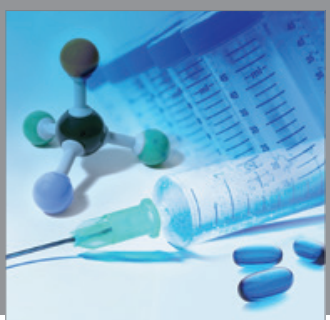

International Journal of

Medicinal Chemistry

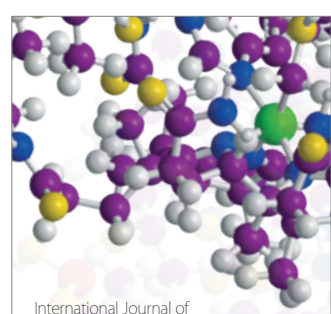

Carbohydrate Chemistry

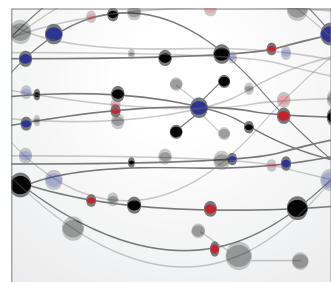

The Scientific World Journal
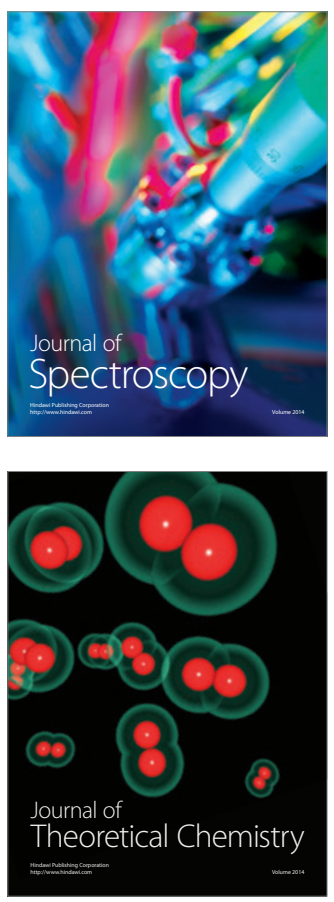
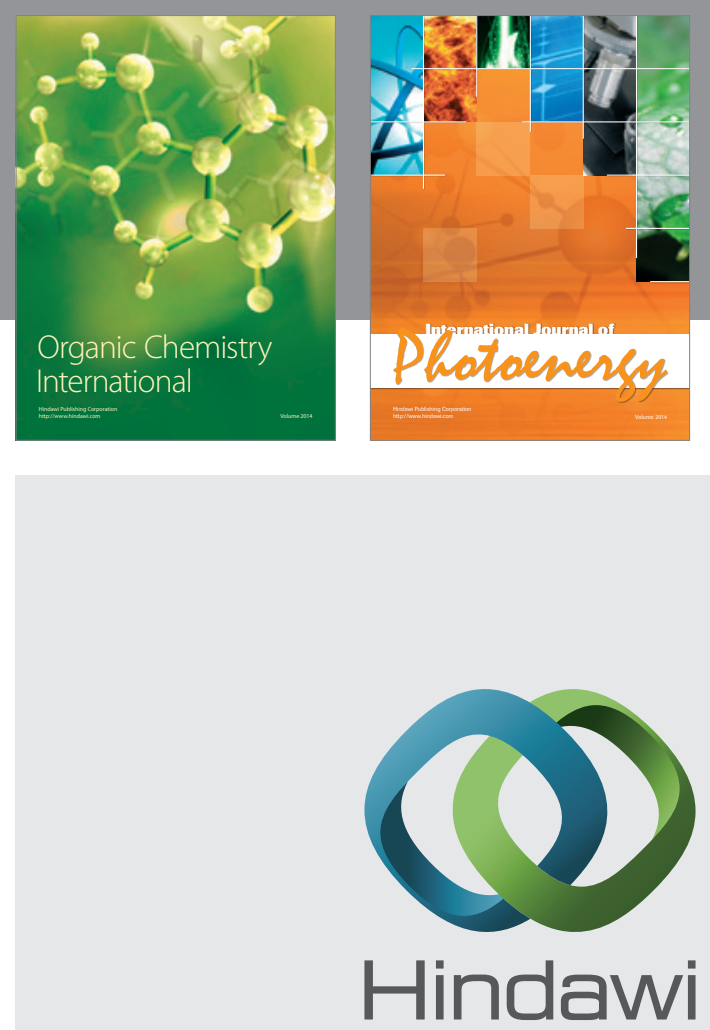

Submit your manuscripts at

http://www.hindawi.com
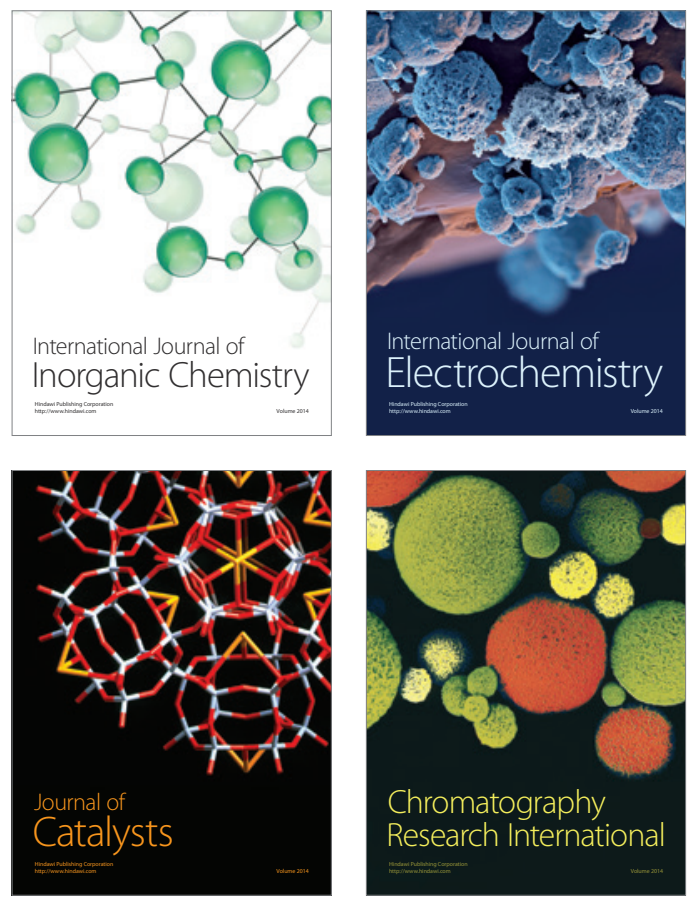
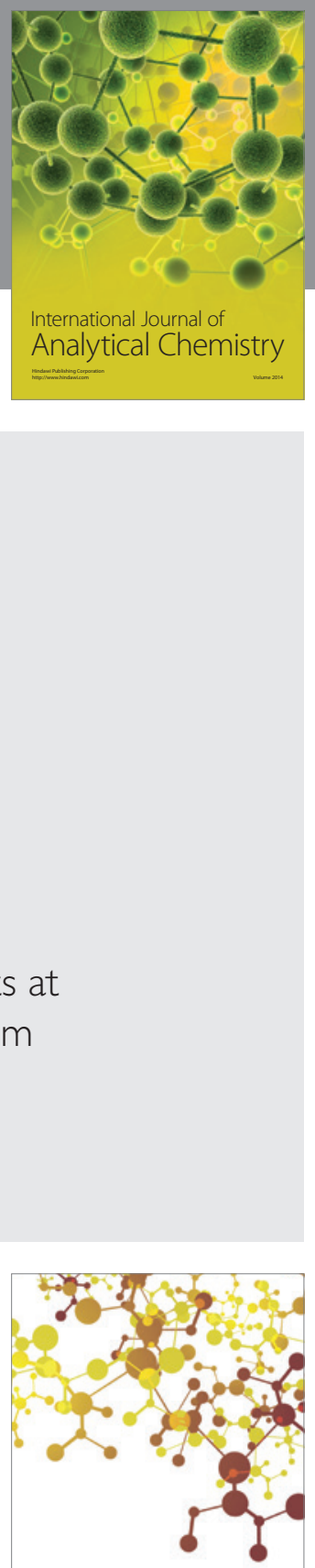

Journal of

Applied Chemistry
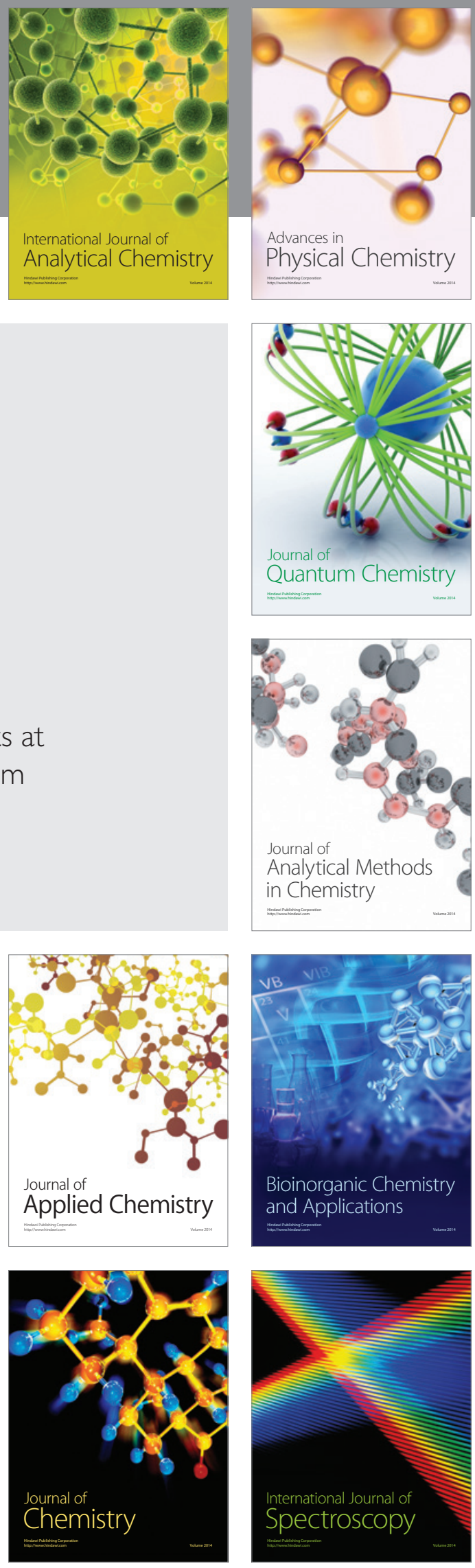